\title{
Editorial
}

\section{Olovnikov's clock: telomeres and vascular biology}

The proliferation, migration, and death of vascular endothelial and smooth muscle cells are crucial to the development of atherosclerosis and its related processes such as postangioplasty restenosis. While a great deal of attention has been paid to many of the factors that influence these events, relatively little attention has been given to one potentially important factor, the age of the cells concerned. Cell age is more appropriately measured in terms of the number of divisions since the cell differentiated from the germ line than in terms of chronological age. Following the work of Hayflick in the 1960s it has been known that virtually all somatic cells in culture go through a finite number of cell divisions and then enter a phase of senescence in which they are no longer susceptible to ordinary mitotic stimuli, and indeed where such stimuli can provoke cell death. ${ }^{12}$ This M1 phase is now thought to be controlled by specific tumour suppressor genes such as p53 and $\mathrm{Rb}^{3-5}$ Cells can be "rescued" from M1 in a variety of ways and in particular by infection with certain transforming viruses-for example, SV40, which work by counteracting the effects of the tumour suppressor genes. Rescued cells go through further cycles of division but nearly all of them will eventually enter a second and lethal phase of senescence called M2. A tiny proportion survive and become immortal in that they continue to proliferate without further signs of senescence in a way indistinguishable from tumour cells.

It is now known, or at least strongly suspected, that the biological clock that determines the effective age of a cell resides in the telomeres. These are the extreme ends of chromosomal DNA, made up of a large number of repeats of a stereotyped and highly conserved nucleotide sequence, TTAGGG. Initial mammalian telomere length is probably of the order of 17000 base pairs. Because of the way in which DNA is replicated there is a statistical risk that a small amount of "end" will be lost at each replication cycle and that the telomeres will become progressively shortened as the cell ages. Olovnikov in 1973 was the first to suggest on theoretical grounds that this was a potential mechanism for a biological clock and this concept has since been supported experimentally. ${ }^{6-8}$ There is good evidence that in tissue culture telomeres shorten progressively with each round of cell division, and human material from subjects of different ages shows a strong age related trend to telomere shortening. Entry to the M2 phase of cell senescence is associated with the presence of very short telomeres, around 1500 base pairs. Cells that become immortal retain short telomeres but acquire the activity of a specific enzyme, telomerase, which is able to repair and extend the telomere to balance the otherwise inevitable shortening at each division. Telomerase activity is present in germ line cells and in many-perhaps all-malignant tumour cells, but is absent from somatic cells. ${ }^{9}{ }^{10}$

The most convincing evidence for telomere involvement in senescence comes from the work of Bodnar and colleagues. ${ }^{11}$ They transfected normal human somatic cells with the gene for the reverse transcriptase component of human telomerase, and showed that its expression rescued cells, including endothelial cells, from senescent behaviour.

There has been much interest in the concept of telomere instability as a factor in the cause of cancer, a condition that increases in incidence as a function of age. ${ }^{12}$ Human telomere shortening as a function of age has been demonstrated in vivo. ${ }^{13}$ Perhaps surprisingly, there has been much less interest in the concept of cell aging as applied to vascular disease, although this is also strongly age related. Of 972 references to telomeres in a MEDLINE literature search from 1993-97, only one referred to vascular tissue or disease. Chang and Harley ${ }^{14}$ found that endothelial telomere length in culture shortened as a function of the number of cell divisions, and that the age related rate of telomere shortening in endothelial cells from iliac arteries was greater than in cells from iliac veins. They also found that rate of telomere loss in DNA from the intima of iliac arteries was greater than for DNA from internal thoracic artery. At first sight the occurrence of replicative aging in vascular cells may appear surprising, as traditionally they have been regarded as cells with a low physiological turnover rate. There is however other independent evidence pointing to senescence, or at least senescence-like behaviour, in vascular cells from elderly patients and from atherosclerotic lesions. Giant endothelial cells with bizarre or multiple nuclei are common in elderly patients, ${ }^{15}{ }^{16}$ and smooth muscle cells isolated from atherosclerotic lesions consistently show a high proportion of cells with a senescent morphology and very limited in vitro replicative capacity. ${ }^{17}$ There are three possible (and not mutually exclusive) explanations for this apparent paradox. First, our traditional methods for assessing cell turnover are inaccurate in vascular tissue or have been incorrectly applied; second, clonal proliferation causes the coexistence of cells of very different replicative age in the same tissue ${ }^{18}{ }^{19}$; and third, there is a short cut to senescence that does not depend on replicative aging, perhaps mediated in some as yet unexplained way by oxidative damage..$^{20}$

The most obvious relevance of cell aging to vascular biology is that the response of an endothelial or smooth muscle cell to either an injury or a growth stimulus is likely to be a function of its age. Stimuli that would in a young endothelial cell be met with a proliferative response might in an old vessel lead to death and endothelial denudation. Similarly in a young artery intimal damage might lead to smooth muscle cell proliferation and migration resulting in a stable cellular plaque, whereas in an old artery the proliferating cells would rapidly senesce and die leading to an acellular and friable intima. Studies on cultured vascular smooth muscle cells indicate that, as in other cell types, p53 and $\mathrm{Rb}$ expression are markers for functional senescence and play an important (but not necessarily exclusive) role in apoptotic death of these cells. ${ }^{21}{ }^{22}$ We do not yet know what drives p53 and $\mathrm{Rb}$ expression - whether this is linked in some way to telomere lengths as suggested by Shay and colleagues, ${ }^{5}$ or whether it is induced by environmental stimuli.

Although viral transformation, and hence release from $\mathrm{Rb}$ mediated replicative control, has been described in animal models, its relevance to human atheroma or restenosis is at present speculative. ${ }^{23}$ Measurement of telomere length may be helpful in understanding how vascular cells behave, particularly in the context of atheroma.

For reasons mentioned above the replicative age of vascular cells in an individual patient do not necessarily 
parallel the patient's chronological age. Chronic vascular stress - for example, as a result of hypertension, might lead to the presence of old cells in a relatively young patient. Conversely, if the Benditts' hypothesis of clonal growth ${ }^{18}$ of an atheromatous plaque is correct, then it would be possible for old and young cells to be present in different parts of the wall of the same vessel. These differences are likely to be relevant-for example, in the choice of vascular interventions. Saphenous vein is a tissue prone to accelerated aging even in its natural position, to judge from the frequency of multinucleate endothelial cells in veins used for grafting (de Bono, unpublished data), and exposure to arterial pressure will further increase cell turnover. It might therefore be a less attractive graft in older patients than the internal thoracic artery. External support ${ }^{24}$ which reduces intimal proliferation, might be even more relevant to an old saphenous vein than to a young one. In angioplasty the age of the intimal tissue would be relevant to the choice of antirestenosis strategy. For example, the effects of local growth factor application is expected to be very different in young and old vessels. ${ }^{25}$

Even more fundamentally, aging may play a central role in atherogenesis. Classically, apoptotic cell death has been regarded as "clean" in that the remnants are ingested by neighbouring cells without exciting an inflammatory reaction. At least as regards vascular smooth muscle cells, the truth of this statement is probably relative rather than absolute. Apoptotic smooth muscle cells have been shown to promote thrombin formation and macrophage activation, ${ }^{26}$ and under circumstances where cell death is affecting more than a small number of cells in a given area it may well induce or sustain a local inflammatory reaction. The concept that atherosclerosis in the average - that is, elderly, human patient is a self perpetuating inflammatory process susceptible to modulation by external factors would be compatible with much recent epidemiological data.

Does the possibility of reversing senescence by the controlled expression of telomerase hold out the promise of more effective treatment of vascular disease? The operative word here would have to be controlled as, at least in our current understanding, telomeric senescence is an important safeguard against neoplasia. If the benefits were worthwhile, they would help to drive the necessary developments in controllable gene expression.

The ancient Greeks believed the three Fates-Atropos, Clotho, and Lachesis-span and cut the thread of life. Substitute telomeric DNA, and we may have come full circle.
Division of Cardiology,

D P DE BONO

Department of Medicine and Therapeutics,

University of Leicester,

Glenfield Hospital, Leicester LE3 9QP, UK

1 Hayflick L, Moorhead PS. The serial cultivation of human diploid cell strains. Exp Cell Res 1961;25:585-621.

2 Hayflick L. The limited in vitro lifetime of human diploid cell strains. Exp Cell Res 1965;37:614-36.

3 Vogelstein B, Kinzler KW. p53 function and dysfunction. Cell 1992;70:523-

4 Shay JW, Pereira Smith OM, Wright WE. A role for both $\mathrm{p} 53$ and Rb in the regulation of cellular senescence Exp Cell Res 1991;196:33-9.

5 Shay JW, Werbin H, Wright WE. Telomere shortening may contribute to ageing and cancer. A perspective. Molecular Cell Differentiation 1994;2:121 .

6 Olovnikov AM. A theory of marginotomy. F Theor Biol 1973;41:181-90.

7 Harley CB, Futcher AB, Greider CW. Telomeres shorten during aging of human fibroblast. Nature (London) 1990;345:458-60.

8 Allsopp RC, Vaziri H, Patterson C, et al. Telomere length predicts replicative capacity of human fibroblasts. Proc Natl Acad Sci USA 1992;89:10114-18.

9 Counter CM, Avilion A, Le Feuvre C, et al. Telomere shortening associated with chromosome instability is arrested in immortal cells which express telwith chromosome instability is arrested in im

$10 \mathrm{Kim}$ NW, Piatyszek MA, Prowse KR, et al. Specific association of human telomerase activity with immortal cells and cancer. Science 1994;266:201114

11 Bodnar AG, Ouellette M, Frolkis M, et al. Extension of lifespan by introduction of telomerase into normal human cells. Science 1998; 279:349-52.

12 Hastie ND, Dempster M, Dunlop MG, et al. Telomere reduction in human colorectal carcinoma and with ageing. Nature (London) 1990;346:866-8.

13 Lindsey J, McGill NI, Lindsey LA, et al. In vivo loss of telomeric repeats with age in humans. Mutation Res 1991;256:45-8.

14 Chang E, Harley CB. Telomere length and replicative aging in human vascular tissue. Proc Natl Acad Sci USA 1995;92:11190-4.

15 Tokunaga O, Fan JL, Watanabe T. Atherosclerosis- and age-related multinucleated variant endothelial cells in primary cullture from human aorta. Am f Pathol 1989; 135:967-76.

16 Burrig KF. The endothelium of advanced arteriosclerotic plaques in humans. Arterioscler Thromb 1991;11:1678-86.

17 Bennett MR, Evan GI, Schwartz SM. Apoptosis of human vascular smooth muscle cells derived from normal vessels and coronary atherosclerotic plaques. $\mathcal{F}$ Clin Invest 1995;95:2266-74.

18 Benditt EP, Benditt JM. Evidence for a monoclonal origin of human atherosclerotic plaques. Proc Natl Acad Sci USA 1973;70:1753-6.

19 Schwartz SM, Majesky MW. Murry CE The intima: development and monoclonal responses to injury. Atherosclerosis 1995;118(suppl):S125-40.

20 de Bono DP, Yang W-D. Exposure to low concentrations of hydrogen peroxide causes delayed endothelial cell death and inhibits proliferation of surviving cells. Atherosclerosis 1995;114:235-45.

21 Bennett MR, Evan GI, Schwartz SM. Apoptosis of rat vascular smooth muscle cells is regulated by p53-dependent and -independent pathways. Circ Res 1995;77:266-73.

22 Katayose D, Wersto R, Cowan $\mathrm{K}$, et al. Consequences of $\mathrm{p} 53$ gene expression by adenovirus vector on cell cycle arrest and apoptosis in human aortic vascular smooth muscle cells. Biochem Biophys Res Comm 1995;215:44651 .

23 Speir E, Modali R, Huang ES, et al. Potential role of human cytomegalovirus and p53 interaction in coronary restenosis. Science 1994;265:391-4.

24 Izzat MB, Mehta D, Bryan AJ, et al. Influence of external stent size on early medial and neointimal thickening in a pig model of saphenous vein bypass grafting. Circulation 1996;94:1741-5.

25 Cascells W. Growth factor therapies for vascular injury and ischemia. Circulation 1995;91:2699-701.

26 Flynn PD, Byrne CD, Baglin TP, et al.Thrombin generation by apoptotic vascular smooth muscle cells. Blood 1997; 89:4378-84. 